\title{
Gain-of-function SOS1 mutations cause a distinctive form of Noonan syndrome
}

Marco Tartaglia ${ }^{1}$, Len A. Pennacchio ${ }^{2,3}$, Chen Zhao ${ }^{4}$, Kamlesh K. Yadav ${ }^{4}$, Valentina Fodale ${ }^{1}$, Anna Sarkozy ${ }^{5}$, Bhaswati Pandit ${ }^{6}$, Kimihiko Oishi $^{6}$, Simone Martinelli ${ }^{1}$, Wendy Schackwitz ${ }^{2,3}$, Anna Ustaszewska $^{2}$, Joel Martin ${ }^{2,3}$, James Bristow ${ }^{2,3}$, Claudio Carta ${ }^{1}$, Francesca Lepri ${ }^{5}$, Cinzia Neri $^{5}$, Isabella Vasta ${ }^{7}$, Kate Gibson ${ }^{8}$, Cynthia J. Curry ${ }^{9}$, Juan Pedro López Siguero ${ }^{10}$, Maria Cristina Digilio $^{11}$, Giuseppe Zampino ${ }^{7}$, Bruno Dallapiccola ${ }^{5}$, Dafna Bar-Sagi ${ }^{4}$, Bruce D. Gelb ${ }^{6}$

${ }^{1}$ Dipartimento di Biologia Cellulare e Neuroscienze, Istituto Superiore di Sanità, Viale Regina Elena, 299, 00161Rome, Italy. ${ }^{2}$ Genomics Division, MS 84-171, Lawrence Berkeley National Laboratory, Berkeley, California 94720 USA. ${ }^{3}$ U.S. Department of Energy Joint Genome Institute, 2800 Mitchell Drive, Walnut Creek, California 94598, USA. ${ }^{4}$ Department of Biochemistry, New York University School of Medicine, 550 First Avenue, New York, New

York 10016. ${ }^{5}$ IRCCS-CSS, San Giovanni Rotondo and CSS-Mendel Institute, Rome, and Department of Experimental Medicine and Pathology, University "La Sapienza”, Viale Regina Elena 261, 00198, Rome, Italy. ${ }^{6}$ Center for Molecular Cardiology and Departments of Pediatrics and Human Genetics, Mount Sinai School of Medicine, One Gustave L. Levy Place, New York,

New York 10029, USA. ${ }^{7}$ Istituto di Clinica Pediatrica, Università Cattolica del Sacro Cuore, Largo A. Gemelli 8, 00168, Rome, Italy. ${ }^{8}$ Royal Children's Hospital, Herston Road, Herston, Queensland 4029, Australia. ${ }^{9}$ Genetic Medicine Central California, 351 East Barstow \#106, Fresno, California 93710, USA. ${ }^{10}$ Endocrinología Pediátrica, Hospital Materno-Infantil, Avida 
Arroyo de los Ángeles, 29011 Málaga, Spain. . ' Genetica Medica, Ospedale "Bambino Gesù", Piazza S. Onofrio 4, 00165, Rome, Italy.

Corresponding Authors:

Marco Tartaglia, Ph.D.

Dipartimento di Biologica Cellulare e Neuroscienze

Istituto di Superiore Sanità

Viale Regina Elena, 299

00161 Rome, Italy

Phone: +39-06-4990-2569

Fax: +39-06-4938-7143

Email: $\underline{\text { mtartaglia@iss.it }}$

Bruce D. Gelb, M.D.

Center for Molecular Cardiology

Mount Sinai School of Medicine

One Gustave L. Levy Place, Box 1040

New York, NY 10029

Tel: $212-241-3302$

Fax: 212-241-3310

Email: bruce.gelb@mssm.edu 
Noonan syndrome (NS) is a developmental disorder characterized by short stature, facial dysmorphia, congenital heart defects and skeletal anomalies ${ }^{1}$. Increased RAS-mitogenactivated protein kinase (MAPK) signaling due to PTPN11 and KRAS mutations cause $50 \%$ of $\mathrm{NS}^{2-6}$. Here, we report that 22 of 129 NS patients without PTPN11 or KRAS mutation (17\%) have missense mutations in SOS1, which encodes a RAS-specific guanine nucleotide exchange factor (GEF). SOS1 mutations cluster at residues implicated in the maintenance of SOS1 in its autoinhibited form and ectopic expression of two NS-associated mutants induced enhanced RAS activation. The phenotype associated with SOS1 defects is distinctive, although within NS spectrum, with a high prevalence of ectodermal abnormalities but generally normal development and linear growth. Our findings implicate for the first time gain-of-function mutations in a RAS GEF in inherited disease and define a new mechanism by which upregulation of the RAS pathway can profoundly change human development.

NS is an autosomal dominant, genetically heterogeneous trait. PTPN11, the first NS-associated gene identified ${ }^{6}$, was discovered through positional cloning. It encodes the non-membranous protein tyrosine phosphatase, SHP-2, that primarily serves positive regulatory roles in signal transduction, particularly via the receptor tyrosine kinase (RTK)-mediated RAS-MAPK pathway. Most mutations perturb the switch between the basally inactive and phosphotyrosinebound active conformations of SHP-2, shifting the equilibrium towards the latter ${ }^{3,6-8}$. Similar somatic PTPN11 mutations underlie several hematopoietic disorders, particularly juvenile myelomonocytic leukemia (JMML) ${ }^{9}$. Gain-of-function RAS mutations and second allele loss of 
NF1 also cause JMML, emphasizing the effects of gain-of-function PTPN11 mutations in increasing RAS-MAPK signaling.

Recent disease gene discovery established that NS and some phenotypically related traits are classed etiologically as disorders of dysregulated RAS-MAPK signaling. LEOPARD syndrome arises from a functionally distinct class of PTPN11 mutations ${ }^{10,11}$. Gain-of-function germline mutations in HRAS have been found in Costello syndrome and in KRAS in severe NS and cardiofacio-cutanteous (CFC) syndrome $\mathrm{e}^{2,4,12,13}$. In addition, BRAF, MEK1 and MEK2 mutations have been observed in $\mathrm{CFC}^{13,14}$.

The initial and rate limiting step in the activation of the RAS-MAPK pathway by extracellular signals is the ligand-dependent conversion of RAS-GDP to RAS-GTP. In the context of RTK signaling, this reaction is catalyzed by the RAS-specific guanine nucleotide exchange factor (GEF) Son of Sevenless (SOS) ${ }^{15}$. Therefore, mutational activation of SOS provides the potential for the upregulation of RAS signaling, an apparent requisite for NS disease pathogenesis. The human genome contains two SOS genes, SOS1 and SOS2, encoding highly similar multi-domain proteins (Fig. 1). Structure function studies of SOS1 have indicated that in the basal state the protein is maintained in an autoinhibited conformation due to complex regulatory intra- and inter-molecular interactions ${ }^{16-18}$. Following RTK stimulation, SOS1 is recruited to the plasma membrane where it acquires a catalytically active conformation by mechanisms that are not fully understood.

To explore whether SOS1 mutations have a role in NS, we assembled genomic DNAs from 96 individuals who were negative for mutations in the two established NS genes (Cohort A) and performed high throughput resequencing of the SOS1 coding region (exons 2-24) and flanking intronic boundaries. We identified 33 sequencing variants, including 12 non-synonymous 
changes observed in 15 samples (Table 1 and Supplemental Table 1). Strikingly, three variants, affecting six subjects, altered $\mathrm{Arg}^{552}$ and a fourth resided nearby, altering Leu ${ }^{550}$. Both residues are evolutionarily conserved.

To provide further evidence that nonsynonymous variants were mutations, we leveraged the sporadic cases. Among the seven variants for which we possessed both parental samples, we failed to observe the relevant sequence change in either parent in five instances; paternity was confirmed in each, providing final proof that these were de novo mutations (Table 1). In single cases, P655L and H1320R were inherited from unaffected parents. W432R, L550P, and Y702H were observed in families with two to three affected individuals and co-segregated with disease. For the exons with variants that were not de novo (exons 11, 13, 14, 17 and 24), we analyzed 155 control individuals, identifying only the P655L variant in the population. Thus, we concluded that ten missense changes were disease-causing mutations. We suspect that the H1320R change is a rare polymorphism but cannot exclude incomplete penetrance in the unaffected parent. The prevalence of SOS1 mutations in the cohort was $13 / 96$ or $12.5 \%$ (95\% C.I.: $7.4-22 \%$ ), which can be considered a lower limit due to the incomplete coverage inherent with our high throughput approach.

To elucidate further the range of molecular defects associated with disease, SOS1 exons 2-24 were scanned with DHPLC in an additional 33 NS samples without PTPN11 or KRAS mutation (Cohort B). Sequencing of amplimers with abnormal denaturing profiles revealed an additional seven missense mutations among nine subjects as well as another probable rare nonsynonymous polymorphism, Q977R, that a proband inherited from an unaffected mother but was not found among the controls (Table 1). 
In Cohort $\mathrm{B}$, two additional mutations altering $\mathrm{Arg}^{552}$ and two independent S548R alleles were observed, providing further evidence for the importance of that region. A second mutation cluster in SOS1's pleckstrin homology $(\mathrm{PH})$ domain became apparent with the identification of an additional instance of $\mathrm{E} 433 \mathrm{~K}$ as well as a $\mathrm{C} 441 \mathrm{Y}$ mutant. A third functional cluster residing in the interacting regions of the Dbl homology (DH) and RAS exchanger motif (Rem) domain was apparent with the identification of M269R, which joined W729L and I733F identified in Cohort A.

The GEF activity of SOS is principally controlled by two regulatory determinants: A catalytic site where the dissociation of nucleotide from RAS occurs and an allosteric site which stimulates exchange activity through the binding of nucleotide-bound $\mathrm{RAS}^{19}$. Whereas the catalytic site is located entirely within the $\operatorname{Cdc} 25$ domain, the allosteric site is bracketed by the $\operatorname{Cdc} 25$ domain and Rem domains. In resting conditions, SOS is autoinhibited due to a blockade of the allosteric site by the DH-PH unit ${ }^{18}$. The three NS-associated SOS1 mutation clusters reside in regions within the molecule that are predicted to contribute structurally to the maintenance of the protein in its autoinhibitied conformation. For example, $\mathrm{Arg}^{552}$ lies in the helical linker between the $\mathrm{PH}$ and Rem domains (Fig. 1) and is predicted to interact directly with the side chains of Asp ${ }^{140}$ and Asp $^{169}$ in the histone domain of $\operatorname{SOS}^{17}{ }^{17}$. The disruption of this interaction could affect the relative orientation of the DH-PH unit and the Rem domain. The mutation cluster represented by E433K and C441Y may disrupt the autoinhibited conformation by causing a structural destabilization of the PH domain. The third cluster (M269R, W729L and I733F) consists of residues that mediate the interaction of the DH and Rem. $\operatorname{Trp}^{729}$ interacts directly with $\mathrm{Met}^{269}$, thereby positioning the DH domain in its autoinhibitory conformation. $\operatorname{Tr}{ }^{729}$ is also critical for the binding of Ras at the allosteric site. Sondermann and co-workers engineered a W729E SOS1 
mutant, which proved unable to bind RAS-GTP at the allosteric site and had severely reduced nucleotide release from RAS-GDP at the catalytic site $^{18}$. Since the NS-associated W729L substitution is more conservative, we suspected that it would preferentially affect autoinhibition.

To examine directly the effects of these mutations on SOS1 function, we chose two representative SOS1 mutants, R552G and W729L, which were expressed transiently in Cos-1 cells. RAS activation, as a read-out of GEF activity, was assessed using a RAF-RBD binding assay (Fig. 2). When wild type SOS1 was expressed, RAS activation was low in starved cells, increased 26-fold rapidly after EGF stimulation and returned to basal levels by $30 \mathrm{~min}$. In contrast, expression of R552G or W729L SOS1 resulted in the accumulation of activated RAS in starved cells and the response to EGF was prolonged. These results confirmed our predictions based on structural information that the NS-associated SOS1 mutations would principally abrogate autoinhibition, resulting in increased RAS activation.

Extensive phenotype data were available for 16 individuals with SOS1 missense mutations. These individuals had cardiac disease (primarily pulmonary valve stenosis), pectus deformities, shorted and webbed neck, and dysmorphic facial features ranging from typical for NS to an appearance resembling cardio-facio-cutaneous syndrome (Table 2 and Supplemental Figure 1). Ectodermal features including facial keratosis pilaris, hypoplastic eyebrows and curly hair were significantly more prevalent among individuals with a SOS1 mutation compared to the general NS population, particularly during childhood. Short stature with a height below the $3^{\text {rd }}$ centile was observed in only 2 of 15 individuals with a SOS1 mutation, whereas prevalence is $70-76 \%$ among NS in general and PTPN11 mutation-negative NS. In contrast, macrocephaly was overrepresented among those with SOS1 mutations. Only one individual with a SOS1 mutation had mental retardation, potentially attributable to critical illness as a newborn. In comparison, 30 
and $35 \%$ of all children with NS and those without a PTPN11 mutation, respectively, require special education.

The analysis of 129 probands with NS from two cohorts identified 14 different molecular lesions among 22 independent cases (17\% of PTPN11-/KRAS-mutation-negative cases), making SOS1 the $2^{\text {nd }}$ most common cause of this disorder thus far. Like PTPN11, SOS1 mutations were found in sporadic and familial NS and engendered a high prevalence of pulmonary valve disease. The SOS1-associated phenotype, while clearly within the NS spectrum, resembled CFC somewhat in its dysmorphia, macrocephaly and ectodermal manifestations but differed notably with preserved development and linear growth. SOS1 mutations were invariably missense defects and clustered at specific regions implicated in the complex regulation of SOS catalytic activity. Among mutations causing developmental disorders with dysregulated RAS-MAPK signaling, SOS1 defects are notable for affecting a protein functioning entirely upstream of RAS.

The results reported here represent the first examples of inherited gain-of-function mutations in SOS1. A frameshift mutation in exon 21 of SOS1 has been reported in one family inheriting hereditary gingival fibromatosis ${ }^{20}$. No additional case inheriting a SOS1 mutation with this autosomal dominant trait has been reported, deferring final proof of causality. Complete loss of Sos1 in mice is embryonic lethal due to placental and cardiac defects, but haploinsufficient mice develop normally ${ }^{21,22}$. Transgenic mice expressing a dominant active form of Sos1 in keratinocytes develop skin papillomata; this epidermal proliferation can be suppressed with reduced Egfr signaling ${ }^{23}$. In contrast with several other genes now associated with inherited disorders with dysregulated RAS signaling, somatic SOS1 mutations have not been reported in cancer. 
The biochemical analysis of two NS-related SOS1 proteins revealed gain-of-function effects resulting in increased RAS activation. Since many of the SOS1 mutations altered residues related to the autoinhibition of SOS, either through interaction of the histone folds with the PHRem linker or interaction of the DH domain at the allosteric RAS binding site, the predominant pathogenetic mechanism appears to be increased availability of the allosteric RAS binding site causing increased GEF activity. This increased GEF activity, in turn, increases RAS activation, which will lead to downstream signaling. SOS proteins also possess GEF activity through their $\mathrm{DH}$ and $\mathrm{PH}$ domains towards Rho GTPases such as $\mathrm{RAC}^{15}$. Aside from genetic evidence implicating RAS signaling in NS and related disorders, the positions of the SOS1 mutations implicate RAS signaling specifically.

The two highly conserved SOS genes in vertebrates are widely expressed ${ }^{24}$. Sos1 and Sos2 bind a docking protein, Grb2, with different affinities ${ }^{25}$ and Sos2 could not compensate for the loss of Sos1 in the Sos1 knockout mice, suggesting that these proteins play unique roles. We examined the hypothesis that SOS2 mutations similar to those in SOS1 can also cause NS but failed to identify sequence changes at homologous positions.

Discovery of disease-causing mutations is challenging for genetic disorders presenting primarily sporadically or in small kindreds. Marked genetic heterogeneity further complicates such efforts due to statistical power issues. Our results provide proof of principal for high throughput resequencing with large cohorts, particularly when the relevant biological pathway can be identified. With three disease genes identified for NS but more than $40 \%$ of cases unexplained, future efforts will be directed towards exploiting this strategy in order to further advance diagnostics and prognostication for this disorder. 


\section{METHODS}

High-Throughput Resequencing of SOS1. We assembled a cohort of 96 human subjects with NS from whom genomic DNAs were obtained from peripheral blood leukocytes. Nearly all subjects were Caucasian and of European ancestry, with the majority being Italian. The subjects did not harbor PTPN11 or KRAS mutation based on scanning of the coding exons with DHPLC (Wave 2100 System, Transgenomic) and/or bidirectional DNA sequencing as previously described $^{2,5}$. For sporadic cases, which represented the vast majority of the subjects, we obtained both parental DNAs whenever possible. All non-anonymous samples were collected under Institutional Review Board-approved protocols and with informed consent.

We chose a cohort of this size with the assumption that SOS1 would account for at least $1 \%$ of NS (or $2 \%$ of PTPN11-/KRAS-negative NS). Based on Collins and Schwartz ${ }^{26}$, this powered the study to detect a mutation in an NS gene at approximately $80 \%$ with $\alpha=0.05$. If the gene accounted for $5 \%$ of PTPN11-/KRAS-negative NS, then the power to detect it with a cohort of this size would exceed $95 \%$.

A high throughput approach to the resequencing of SOS1 was performed at the Joint Genome Institute. The resequencing protocol was as follows: Oligonucleotide primers (sequences available upon request) for amplifying the SOS1 coding exons $(n=23)$ were designed to give a product size in the range of $200-700 \mathrm{bp}$ with a minimum of $40 \mathrm{bp}$ flanking the splice sites using the Exon Primer program (http://ihg.gsf.de/ihg/ExonPrimer.html), which is bundled with the UCSC Genome Browser (hg17 genome build: http://genome.ucsc.edu/). M13F and M13R tags were added to the forward and reverse primers, respectively. Five nanograms of genomic DNA from each NS sample was amplified in a $8 \mu \mathrm{l}$ PCR reaction using AmpliTaq Gold (Applied 
Biosystems) using PE 9700 machines and subsequently cleaned using a diluted version of the Exo-SAP based PCR product pre-sequencing kit (USB Corporation) dispensed by a nanoliter dispenser (Deerac Fluidics Equator). All PCR set-up procedures were performed in a 384-well format using a Biomek NX workstation following their optimization. Sequencing reactions were then performed using the M13 primers along with BigDye Terminator v3.1 Cycle Sequencing Kit (Applied Biosystems) and cleaned with BET before separation on an ABI 3730xl DNA Analyzer. Base calling, quality assessment and assembly were carried out using the Phred, Phrap, Polyphred, Consed software suite (http://www.phrap.org/). All sequence variants identified were verified by manual inspection of the chromatograms and putative causative mutations were verified using another independent sequencing reaction.

When available, parental DNAs were then sequenced to establish whether the change was de novo. Paternity was confirmed by simple tandem repeat (STR) genotyping using the AmpF/STR Identifier PCR Amplification Kit (Applied Biosystems). Anonymous Caucasian control genomic DNAs were screened for SOS1 coding exons in which putative mutations had been identified using DHPLC analysis of PCR-generated amplimers at column temperatures recommended by the Navigator version 1.5.4.23 software (oligonucleotide primer sequences and DHPLC conditions available upon request). Amplimers having abnormal denaturing profiles were purified (Microcon PCR, Millipore) and sequenced bi-directionally using the ABI BigDye terminator Sequencing Kit v.1.1 (Applied Biosystems) and an ABI Prism 310 Genetic Analyzer (Applied Biosystems). Eighty-five additional Caucasian control DNAs were digested with MneI (New England Biolabs) or BsrsI (Promega) to further exclude occurrence of the $1297 \mathrm{G} \rightarrow \mathrm{A}$ and $1649 \mathrm{~T} \rightarrow \mathrm{C}$ missense changes, respectively. Informatics analysis of sequences to predict splice acceptor and donor sites as well as exonic splice enhancers was performed using programs 
available at the following websites: http://www.cbs.dtu.dk/services/NetGene2, http://www.fruitfly.org/seqtools/splice.html, http://rulai.cshl.edu/ tools/ESE.

Analysis of the Second NS Cohort. We assembled a $2^{\text {nd }}$ panel of 33 PTPN11-negative/KRASnegative NS genomic DNAs. This panel was used as confirmatory of the results of the $1^{\text {st }}$ panel and to extend the range of mutations associated with NS. These DNAs were scanned for SOS1 mutations using DHPLC and abnormal amplimers were sequenced bi-directionally as described above.

RAS Activation Assay. GST-RAF-RBD fusion proteins were expressed in Escherichia coli by induction with $0.5 \mathrm{mM}$ of isopropyl-1-thio- $\beta$-D-galactopyranoside (IPTG) for 5 hours. The expressed fusion proteins were isolated from bacteria lysates by affinity chromatography with glutathione agarose beads for $1 \mathrm{~h}$ at $4{ }^{\circ} \mathrm{C}$. Cos- 1 cells were co-transfected with HA-tagged RAS wild type and wild type (WT) or mutant SOS1. Twenty-four hours after transfection, cells were switched to serum-starvation medium (0\% DMEM) for $16 \mathrm{~h}$. Following stimulation with EGF (10 ng/ml) for the indicated intervals at $37{ }^{\circ} \mathrm{C}$, the cells were collected in RBD lysis buffer containing 25mM Tris- $\mathrm{HCl}$ (pH7.4), $120 \mathrm{mM} \mathrm{NaCl}, 10 \mathrm{mM} \mathrm{MgCl}, 1 \mathrm{mM}$ EDTA, 10\% glycerol, $10 \mathrm{mg} / \mathrm{ml}$ pepstatin, $50 \mathrm{mM} \mathrm{NaF}, 1 \%$ aprotinin, $10 \mu \mathrm{g} / \mathrm{ml}$ leupeptin, $1 \mathrm{mM} \mathrm{Na} \mathrm{VO}_{4}, 10 \mathrm{mM}$ benzamidine, $10 \mu \mathrm{g} / \mathrm{ml}$ soybean trypsin inhibitor, $1 \%$ NP40, $0.25 \%$ sodium deoxycholic acid. For each condition, $400 \mu \mathrm{g}$ of whole cell lysate was pre-cleared with $10 \mu \mathrm{l} 50 \% \mathrm{GST}$ for $5 \mathrm{~min}$ at $4{ }^{\circ} \mathrm{C}$. The samples were then centrifuged and supernatants were transferred to Eppendorf tubes containing $20 \mu \mathrm{g}$ GST-RAF-RBD immobilized beads. Samples were incubated for $1.5 \mathrm{~h}$ at $4{ }^{\circ} \mathrm{C}$. The complexes were collected by centrifugation and washed six times with buffer containing 25 
$\mathrm{mM}$ Tris- $\mathrm{HCl}$ (pH 7.4), $120 \mathrm{mM} \mathrm{NaCl}, 10 \mathrm{mM} \mathrm{MgCl} 2,1 \mathrm{mM}$ EDTA, $10 \%$ glycerol, $50 \mathrm{mM}$ NaF, $1 \%$ NP40. Protein complexes were eluted with SDS sample buffer, separated by SDS12.5\% PAGE, and transferred to nitrocellulose membrane. The proteins were detected by Western blot with anti-HA antibody $(12 \mathrm{CA} 5 ; 1: 10,000)$ and goat anti-mouse HRP conjugated secondary antibody (Cappel; 1:10,000).

\section{Acknowledgments}

We are indebted to the patients and families who participated in the study, the physicians who referred the subjects, and the Joint Genome Institute's production sequencing group. This work was supported by Telethon-Italy grant GGP04172 and "Programma di Collaborazione ItaliaUSA/malattie rare" (M.T.), US National Institutes of Health Grants HL71207, HD01294, and HL074728 (B.D.G.), CA55360 and CA28146 (D.B.-S.), and Italian Ministry of Health Grant RC 2006 (B.D.). Research conducted at the E.O. Lawrence Berkeley National Laboratory and the Joint Genome Institute was performed under Department of Energy Contract DEAC0378SF00098, University of California (LAP). 
Table 1: Missense Variants

\begin{tabular}{|c|c|c|c|c|c|}
\hline Exon & $\begin{array}{c}\text { DNA } \\
\text { Sequence } \\
\text { Variant } \\
\end{array}$ & $\begin{array}{l}\text { Amino Acid } \\
\text { Substitution }\end{array}$ & $\begin{array}{c}\text { SOS1 } \\
\text { Domain }\end{array}$ & $\begin{array}{c}\text { Cohort } \\
\text { (Observations) }\end{array}$ & $\begin{array}{c}\text { Mut/Poly; } \\
\text { Basis }^{1}\end{array}$ \\
\hline 11 & $1294 \mathrm{~T} \rightarrow \mathrm{C}$ & W432R & $\mathrm{PH}$ & $\mathrm{A}(1)$ & Mut; Ctrl \\
\hline 11 & $1297 \mathrm{G} \rightarrow \mathrm{A}$ & $\mathrm{E} 433 \mathrm{~K}$ & $\mathrm{PH}$ & A (1) & Mut; Ctrl \\
\hline 11 & $1649 \mathrm{~T} \rightarrow \mathrm{C}$ & L550P & $\begin{array}{l}\text { PH-Rem } \\
\text { Linker }\end{array}$ & $\mathrm{A}(1)$ & Mut; Ctrl \\
\hline 11 & $1654 \mathrm{~A} \rightarrow \mathrm{G}$ & R552G & $\begin{array}{l}\text { PH-Rem } \\
\text { Linker }\end{array}$ & A (4) & Mut; DN \\
\hline 11 & $1655 \mathrm{G} \rightarrow \mathrm{A}$ & R552K & $\begin{array}{l}\text { PH-Rem } \\
\text { Linker }\end{array}$ & A (1) & Mut; DN \\
\hline 11 & $1656 \mathrm{G} \rightarrow \mathrm{C}$ & R552S & $\begin{array}{l}\text { PH-Rem } \\
\text { Linker }\end{array}$ & A (1) & Mut; DN \\
\hline 13 & $1964 \mathrm{C} \rightarrow \mathrm{T}$ & P655L & Rem & A (1) & Poly \\
\hline 14 & $2104 \mathrm{~T} \rightarrow \mathrm{C}$ & Y702H & Rem & A (1) & Mut; Ctrl \\
\hline 15 & $2186 \mathrm{G} \rightarrow \mathrm{T}$ & W729L & Rem & A (1) & Mut; DN \\
\hline 15 & $2197 \mathrm{~A} \rightarrow \mathrm{T}$ & $\mathrm{I} 733 \mathrm{~F}$ & Rem & $A(1)$ & Mut; DN \\
\hline 17 & $2536 \mathrm{G} \rightarrow \mathrm{A}$ & E846K & $\mathrm{Cdc} 25$ & A (1) & Mut; Ctrl \\
\hline 24 & $3959 \mathrm{~A} \rightarrow \mathrm{G}$ & H1320R & C-Term & A (1) & Poly \\
\hline 4 & $322 \mathrm{G} \rightarrow \mathrm{A}$ & E108K & $\mathrm{HF}$ & B (2) & Mut; Ctrl \\
\hline 7 & $806 \mathrm{~T} \rightarrow \mathrm{G}$ & M269R & $\mathrm{DH}$ & B (1) & Mut; DN \\
\hline 11 & $1297 \mathrm{G} \rightarrow \mathrm{A}$ & E433K & PH & B (1) & Mut; Ctrl \\
\hline 11 & $1322 \mathrm{G} \rightarrow \mathrm{A}$ & C441Y & $\mathrm{PH}$ & B (1) & Mut; DN \\
\hline 11 & $1642 \mathrm{~A} \rightarrow \mathrm{C}$ & S548R & $\begin{array}{l}\text { PH-Rem } \\
\text { Linker }\end{array}$ & B (2) & Mut; DN \\
\hline 11 & $1654 \mathrm{~A} \rightarrow \mathrm{G}$ & R552G & $\begin{array}{l}\text { PH-Rem } \\
\text { Linker }\end{array}$ & B (1) & Mut; DN \\
\hline 11 & $1656 \mathrm{G} \rightarrow \mathrm{C}$ & R552S & $\begin{array}{l}\text { PH-Rem } \\
\text { Linker }\end{array}$ & B (1) & Mut; DN \\
\hline 19 & $2930 \mathrm{~A} \rightarrow \mathrm{T}$ & Q977R & $\mathrm{Cdc} 25$ & B (1) & Poly \\
\hline
\end{tabular}

${ }^{1}$ Ctrl- Controls; DN- de novo 
Table 2: Genotype-Phenotype Correlation

\begin{tabular}{|c|c|c|c|}
\hline \multirow[b]{2}{*}{ Clinical Feature } & \multicolumn{3}{|c|}{ No./Total (\%) of Subjects } \\
\hline & $\begin{array}{l}\text { SOS1 } \\
\text { Mutation }\end{array}$ & All $^{\mathrm{a}}$ & $\begin{array}{c}\text { Without PTPN11 } \\
\text { Mutation b }^{\mathbf{b}}\end{array}$ \\
\hline Polyhydramnios & $8 / 15(53)$ & $43 / 130(33)$ & NA \\
\hline Fetal Macrosomia & $9 / 15(60)$ & NA & NA \\
\hline $\begin{array}{l}\text { Short Stature }\left(<3^{\text {rd }}\right. \\
\text { centile })\end{array}$ & 2/15 (13) & $84 / 115(73)^{* * *}$ & $45 / 64(70)^{* * *}$ \\
\hline Macrocephaly & $9 / 16(56)$ & $19 / 151(12)^{* * *}$ & NA \\
\hline $\begin{array}{l}\text { Downslanting Palpebral } \\
\text { Fissures }\end{array}$ & 15/16 (94) & NA & NA \\
\hline Ptosis & $16 / 16(100)$ & NA & NA \\
\hline $\begin{array}{l}\text { Low-Set Ears with } \\
\text { Thickened Helix }\end{array}$ & $16 / 16(100)$ & NA & NA \\
\hline Thick Lips/Macrostomia & $14 / 16(88)$ & NA & NA \\
\hline Short/Webbed Neck & $15 / 16(94)$ & NA & NA \\
\hline Abnormal Pectus & $16 / 16(100)$ & 144/151 (95) & $46 / 61(75)^{*}$ \\
\hline Cardiac Involvement & $13 / 16(81)$ & 132/151 (87) & $42 / 66(64)$ \\
\hline $\begin{array}{l}\text { Pulmonary Valve } \\
\text { Stenosis }\end{array}$ & $10 / 16(62)$ & 93/151 (62) & $30 / 65(46)$ \\
\hline Septal Defect & $4 / 16(25)$ & 29/151 (19) & $11 / 63(18)$ \\
\hline $\mathrm{HCM}$ & $2 / 16(12)$ & $30 / 151(20)$ & $17 / 65(26)$ \\
\hline Facial Keratosis Pilaris & $8 / 16(50)$ & $21 / 151(14)^{* * *}$ & NA \\
\hline Curly Hair & $14 / 16(88)$ & $44 / 151(29)^{* * *}$ & NA \\
\hline Cryptorchidism & 6/9 (67) & $64 / 83(77)$ & $25 / 35(71)$ \\
\hline Mental Retardation & $1 / 16(6)$ & $32 / 105(30)^{*}$ & $21 / 59(36)^{*}$ \\
\hline Bleeding Diathesis & $5 / 16(31)$ & $37 / 151(25)$ & NA \\
\hline
\end{tabular}

${ }^{\mathrm{a}}$ From Ref. ${ }^{27}$. ${ }^{\mathrm{b}}$ From Ref. 5. Significance: $*,<.05 ; * *,<.01 ; * * *,<.001$. Definitions: HCM, hypertrophic cardiomyopathy; NA, not available. 


\section{References}

1. Noonan, J.A. Hypertelorism with Turner phenotype. A new syndrome with associated congenital heart disease. Am J Dis Child 116, 373-80 (1968).

2. Carta, C. et al. Germline missense mutations affecting KRAS isoform B are associated with a severe Noonan syndrome phenotype. Am J Hum Genet 79, 129-135 (2006).

3. Fragale, A., Tartaglia, M., Wu, J. \& Gelb, B.D. Noonan syndrome-associated SHP2/PTPN11 mutants cause EGF-dependent prolonged GAB1 binding and sustained ERK2/MAPK1 activation. Hum Mutat 23, 267-77 (2004).

4. Schubbert, S. et al. Germline KRAS mutations cause Noonan syndrome. Nat Genet 38, 331-6 (2006).

5. Tartaglia, M. et al. PTPN11 mutations in Noonan syndrome: molecular spectrum, genotype-phenotype correlation, and phenotypic heterogeneity. Am J Hum Genet 70, 1555-63 (2002).

6. Tartaglia, M. et al. Mutations in PTPN11, encoding the protein tyrosine phosphatase SHP-2, cause Noonan syndrome. Nat Genet 29, 465-8 (2001).

7. Keilhack, H., David, F.S., McGregor, M., Cantley, L.C. \& Neel, B.G. Diverse biochemical properties of Shp2 mutants. Implications for disease phenotypes. J Biol Chem 280, 30984-93 (2005).

8. Tartaglia, M. et al. Diversity and functional consequences of germline and somatic PTPN11 mutations in human disease. Am J Hum Genet 78, 279-90 (2006).

9. Tartaglia, M. et al. Somatic mutations in PTPN11 in juvenile myelomonocytic leukemia, myelodysplastic syndromes and acute myeloid leukemia. Nat Genet 34, 148-50 (2003). 
10. Digilio, M.C. et al. Grouping of Multiple-Lentigines/LEOPARD and Noonan Syndromes on the PTPN11 Gene. Am J Hum Genet 71, 389-94 (2002).

11. Legius, E. et al. PTPN11 mutations in LEOPARD syndrome. J Med Genet 39, 571-4 (2002).

12. Aoki, Y. et al. Germline mutations in HRAS proto-oncogene cause Costello syndrome. Nat Genet 37, 1038-40 (2005).

13. Niihori, T. et al. Germline KRAS and BRAF mutations in cardio-facio-cutaneous syndrome. Nat Genet 38, 294-6 (2006).

14. Rodriguez-Viciana, P. et al. Germline mutations in genes within the MAPK pathway cause cardio-facio-cutaneous syndrome. Science 311, 1287-90 (2006).

15. Nimnual, A. \& Bar-Sagi, D. The two hats of SOS. Sci STKE 2002, PE36 (2002).

16. Corbalan-Garcia, S., Margarit, S.M., Galron, D., Yang, S.S. \& Bar-Sagi, D. Regulation of Sos activity by intramolecular interactions. Mol Cell Biol 18, 880-6 (1998).

17. Sondermann, H., Nagar, B., Bar-Sagi, D. \& Kuriyan, J. Computational docking and solution x-ray scattering predict a membrane-interacting role for the histone domain of the Ras activator son of sevenless. Proc Natl Acad Sci U S A 102, 16632-7 (2005).

18. Sondermann, H. et al. Structural analysis of autoinhibition in the Ras activator Son of sevenless. Cell 119, 393-405 (2004).

19. Margarit, S.M. et al. Structural evidence for feedback activation by Ras.GTP of the Rasspecific nucleotide exchange factor SOS. Cell 112, 685-95 (2003).

20. Hart, T.C. et al. A mutation in the SOS1 gene causes hereditary gingival fibromatosis type 1. Am J Hum Genet 70, 943-54 (2002). 
21. Qian, X. et al. The Sos1 and Sos2 Ras-specific exchange factors: differences in placental expression and signaling properties. Embo $J$ 19, 642-54 (2000).

22. Wang, D.Z. et al. Mutation in Sos1 dominantly enhances a weak allele of the EGFR, demonstrating a requirement for Sos1 in EGFR signaling and development. Genes Dev 11, 309-20 (1997).

23. Sibilia, M. et al. The EGF receptor provides an essential survival signal for SOSdependent skin tumor development. Cell 102, 211-20 (2000).

24. Bowtell, D., Fu, P., Simon, M. \& Senior, P. Identification of murine homologues of the Drosophila son of sevenless gene: potential activators of ras. Proc Natl Acad Sci U S A 89, 6511-5 (1992).

25. Yang, S.S., Van Aelst, L. \& Bar-Sagi, D. Differential interactions of human Sos1 and Sos2 with Grb2. J Biol Chem 270, 18212-5 (1995).

26. Collins, J.S. \& Schwartz, C.E. Detecting polymorphisms and mutations in candidate genes. Am J Hum Genet 71, 1251-2 (2002).

27. Sharland, M., Burch, M., McKenna, W.M. \& Paton, M.A. A clinical study of Noonan syndrome. Arch Dis Child 67, 178-83 (1992). 


\section{Figure Legends}

Figure 1 (a) SOS1 domain structure and location of residues altered in Noonan syndrome. The predicted amino acid substitutions from the 14 SOS1 missense mutations are positioned below the cartoon of the SOS1 protein with its functional domains indicated above. Abbreviations: DH, Dbl homology domain; PH, plekstrin homology domain; Rem, RAS exchanger motif. (b) Location of the mutated residues on the three-dimensional structure of SOS1. The functional domains are color coded as follows: Histone folds, cyan; DH, magenta; PH, orange; Rem, green; Cdc25, yellow. Residues affected by mutations are indicated with their lateral chains and numbered. Based on Ref. 17, which utilized structural data and computational modeling.

Figure 2 RAS activation assay. Full-length, HA-tagged wild type (WT), R552G or W729L SOS1 were expressed in Cos-1 cells with HA-RAS. Binding of RAS to RAF-RBD was assayed to assess RAS activation in serum-starved cells ( 0 min) and after 5, 15 and 30 min of EGF stimulation. Total RAS and SOS1 proteins in the whole cell lysates (WCL), shown in the lower two panels, and activated RAS, upper panel, were detected immunologically with anti-HA. All fold activation ratios were compared to SOS-WT at $0 \mathrm{~min}$.

Supplementary Figure 1 Facial dysmorphia in SOS1-associated Noonan syndrome. Photographs of 10 individuals with Noonan syndrome and the SOS1 mutation indicated below each of them. 
Histone

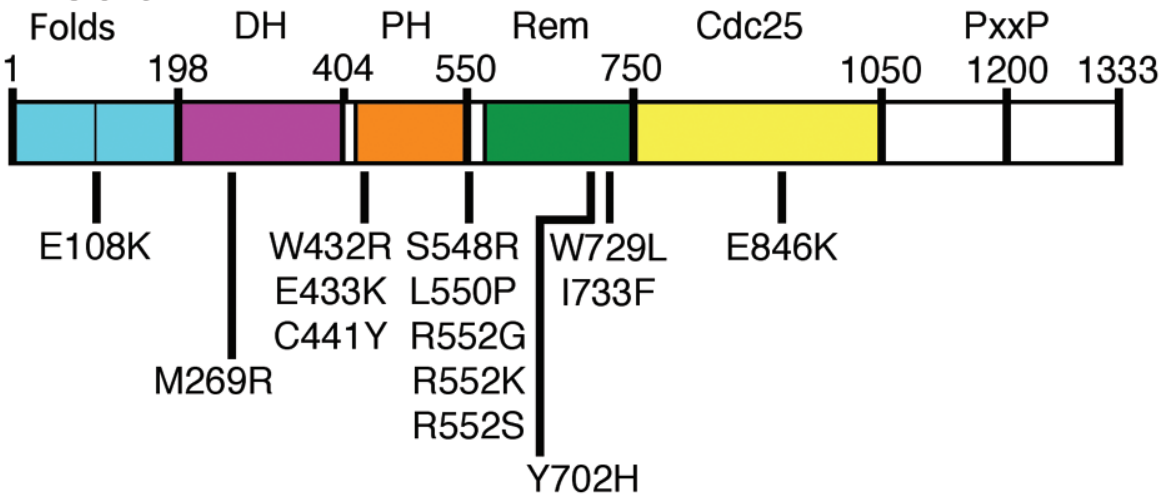




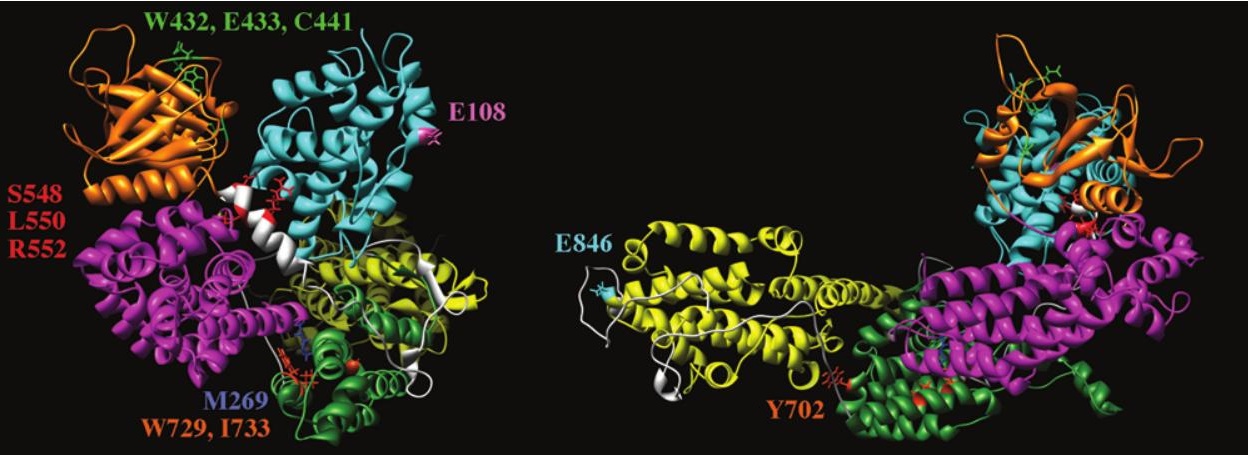


$\begin{array}{lllllllllllll}\text { EGF(min): } & \mathbf{0} & \mathbf{5} & \mathbf{1 5} & \mathbf{3 0} & \mathbf{0} & \mathbf{5} & \mathbf{1 5} & \mathbf{3 0} & \mathbf{0} & \mathbf{5} & \mathbf{1 5} & \mathbf{3 0}\end{array}$

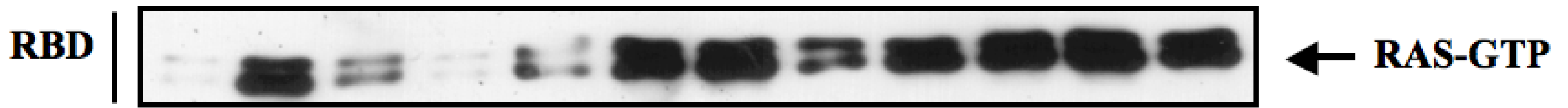

$\begin{array}{lllllllllllll}\text { Fold Activation: } & 1 & 26 & 8 & 1 & 11 & 30 & 33 & 18 & 24 & 27 & 30 & 27\end{array}$

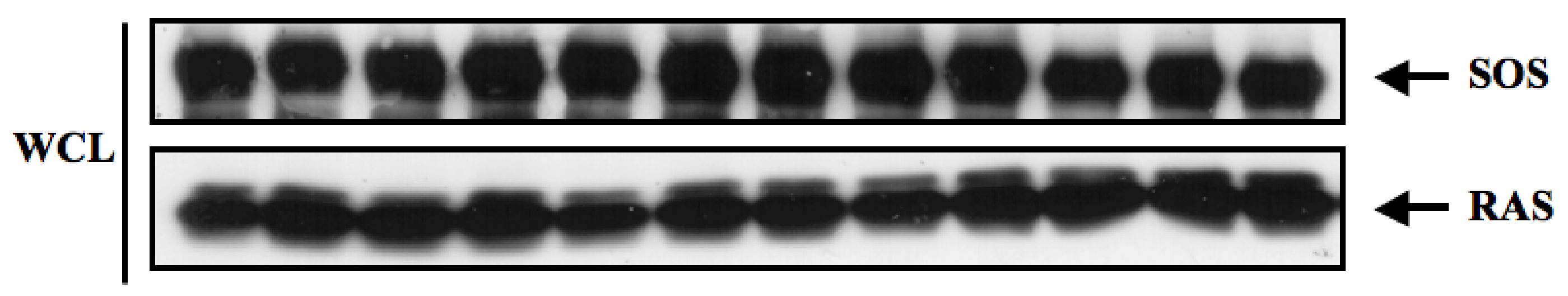

IB: anti-HA 


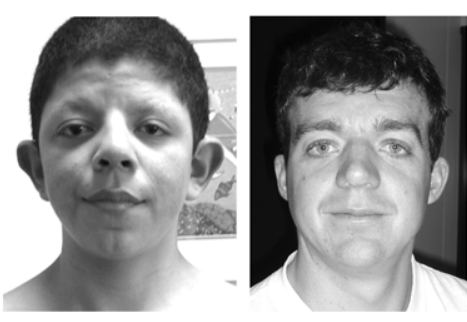

E108K

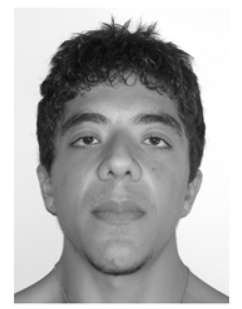

R552G

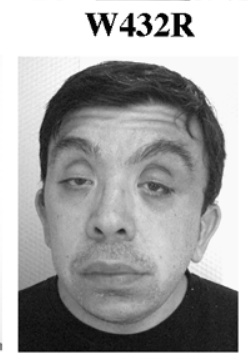

R552G

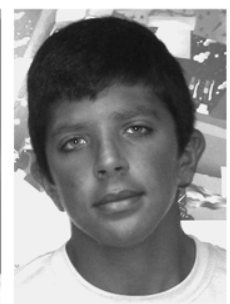

E433K

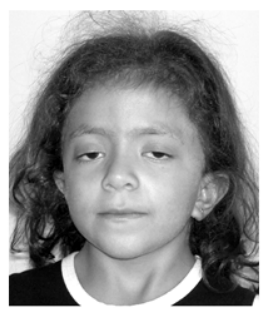

R552K

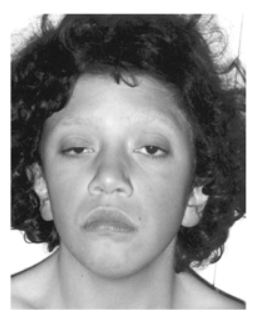

C441Y

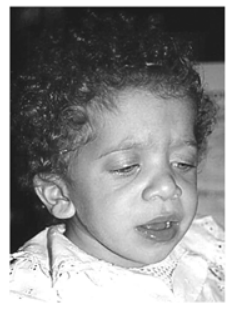

R552S

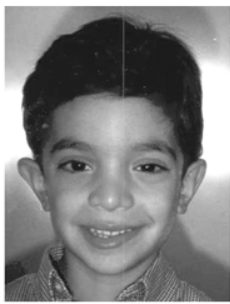

S548R

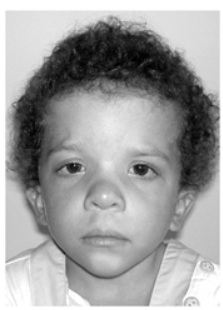

I733F 